\title{
A Stochastic Model for BitTorrent-like Systems
}

\author{
Pei Li \\ Department of Computer \\ Science \& Technology \\ University of Science \& \\ Technology of China \\ lipei@mail.ustc.edu.cn
}

\author{
John C.S. Lui \\ Department of Computer \\ Science \& Engineering \\ The Chinese University of \\ Hong Kong \\ cslui@cse.cuhk.edu.hk
}

\author{
Yinlong Xu \\ Department of Computer \\ Science \& Technology \\ University of Science \& \\ Technology of China \\ ylxu@ustc.edu.cn
}

\section{INTRODUCTION}

BitTorrent (BT) [1] is a P2P protocol designed for fast and efficient distribution of large files. Many factors contribute to the BT system dynamics, such as the arrival and departure of peers, and the terminations of connections when peers find better neighbors. Qiu and Srikant [6] consider the arrival and departure process as the main source of dynamics. Liao et al. [3] study the connection dynamics, in particular, they consider the effect of optimistic unchoking and tit-for-tat, and estimate the average download rates for heterogeneous peers. However, as far as we know, no work has considered these two types of dynamics together.

This paper takes into account these dynamics, and presents a stochastic model to study the fractions of peers with different connection numbers, from which we can compute the average number of connections per peer. In practice, to limit P2P applications, some ISPs charge users by the numbers of TCP connections, so it is important to estimate the average number of connections per peer. Using this model, BT users can estimate the average number of connections per peer, and then choose the numbers of TCP connections to minimize their costs. Since we assume homogeneous peers, the average download rate is equal to the upload rate, so it is trivial to estimate the average download rate or the average file download time in this model.

\subsection{Peers}

We assume homogeneous peers here, and each peer has an upload capacity of $C$. Then the average download rate is also $C$. The maximum number of uploads (or connections) is $N \geq 1$. Peers are classified into different types according to the numbers of active uploads connections, i.e., a peer maintaining $i$ uploads is of type $i$. Note that there are no peers of type 0 , since each peer always maintains an upload via optimistic unchoking. For peers of type $i$, the bandwidth per upload is $c_{i}=C / i$. We denote the fraction of peers of type $i$ in the system as $q_{i}$, with $\sum_{1<i<N} q_{i}=1$. We assume the download capacity is not the system bottleneck, and the limit to the system throughput is the peer upload capacity, which is a well-known assumption suggested in $[5,2,4]$.

\subsection{Optimistic Unchoking and Tit-for-tat}

Permission to make digital or hard copies of all or part of this work for personal or classroom use is granted without fee provided that copies are not made or distributed for profit or commercial advantage and that copies bear this notice and the full citation on the first page. To copy otherwise, to republish, to post on servers or to redistribute to lists, requires prior specific permission and/or a fee.

Copyright 2008 ACM MAMA Workshop .
Optimistic unchoking and tit-for-tat are considered here. The download rate of a peer is influenced by these two mechanisms, and we denote the download rate caused by optimistic unchoking (tit-for-tat) as $\bar{d}^{O U}\left(\bar{d}^{T F^{T}}\right)$. Then the average download rate is $\bar{d}=\bar{d}^{O U}+\bar{d}^{T F T}$. Since the receiver of optimistic unchoking is randomly selected, a peer has equal probability to receive an optimistic unchoking upload from all other peers, so $\bar{d}^{O U}=$ $\sum_{i=1}^{N} c_{i} q_{i}$, where we know $\bar{d}^{O U}$ is the same for all peers.

When an optimistic unchoking occurs, the receiver should decide whether to upload to the sender subjected to the tit-for-tat mechanism. To study this influence, we model the optimistic unchoking and tit-for-tat as the following processes: (1) Random upload: It is used to characterize optimistic unchoking. Every peer maintains a random upload, whose receiver is randomly selected. The duration is the same as optimistic unchoking, and $\bar{d}^{O U}$ is the average rate of the random upload. (2) Random request: It is used to characterize the tit-for-tat process. When a random request arrives, a peer selects another peer randomly, and sends the later a request. If both peers find it beneficial to exchange service, a bidirectional connection is established.

In a realistic BT system, at the end of each random upload, there should be a random request. To simplify the analysis, we assume these two processes are independent. Then peers are memoryless and all connections except optimistic unchoking are bidirectional. In the rest of this paper, unless we state otherwise, we will use "connection" instead of "bidirectional connection" for simplicity.

\subsection{System Dynamics}

In a realistic BT system, connections may be terminated due to the following reasons: 1) peers finish the file download and leave the system, and 2) for a peer, if the number of connections reaches the maximum and it finds a non-neighboring peer with a higher bandwidth per upload, it terminates the connection with the minimum bandwidth download so as to establish a new one with the non-neighboring peer.

Assume the peer arrival process is a Poisson process with rate $\lambda$, and peers will leave the system immediately after they finish the file download. If the file size is $F$ and the average download rate of type $i$ peers is $\bar{d}_{i}$, then the departure rate for a single type $i$ peer is $\mu_{i}=\bar{d}_{i} / F$. Since the average download time of all peers is $F / C$, using the Little's Theorem, the number of peers in the system at steady state is $M=\lambda \cdot F / C$.

If there is a connection between a type $i$ peer and a type $j$ peer, we denote this connection as $i \leftrightarrow j$, and $\rho(i, j)$ is the rate that the type $j$ peer terminates this connection. We have

$$
\rho(i, j) \geq \mu_{j} .
$$

Assume the arrival of random requests is a Poisson process, and the duration of an optimistic unchoking is $1 / \omega$ in a BT system. 
Note that for each optimistic unchoking, there should be a random request. So the rate of a peer sending random requests is $\omega$, which is also the rate of a peer receiving random requests.

\section{A STOCHASTIC MODEL}

In this section, we define peer state, and characterize the system state by the numbers of peers in different peer states. Then we express the transition rates between different system states, and solve a system of differential equations to get the fractions of peers in different peer states at steady state. We first give some definitions and notations:

(1) Peer state: We say a peer is in peer state $s=\left(n_{1}, n_{2}, \cdots, n_{N}\right)$, where $n_{i}$ is the number of its type $i$ neighbors. We introduce the definition of peer state, because peers of the same type may behave differently since they may have different types of neighbors, so one cannot simply use peer type to describe the system accurately.

(2) Feasible peer state: We denote $\mathcal{S}$ as the feasible peer state set. In this model, we fix the number of optimistic unchoking connections to 1 , and consider the dynamics of tit-for-tat connections only. Then the maximum number of connections a peer can maintain is $N-1$. Denote $I_{k}$ as the vector whose first $k$ coordinates equal 1 and other coordinates equal zero. For each $s \in \mathcal{S}, I_{N} \cdot s^{T} \leq N-1$, $n_{1}=0$ and $n_{i} \geq 0$. Let $\mathcal{S}_{i}$ be the set of peer states $s \in \mathcal{S}$ where $I_{N} s^{T}=i-1$. Then all peers in peer state $s \in \mathcal{S}_{i}$ are of type $i$.

(3) Exogenous peer arrival rate and exogenous peer departure rate: Let $\lambda(s)$ be the exogenous peer arrival rate of peer state $s$. Note that $\lambda(s)$ is zero except for the peer state $(0,0, \cdots, 0)$. Denote $E_{i}$ as the vector whose $i$ th coordinate equals 1 and all other equal zero. For peers in peer state $s$, the average download rate is $\bar{d}(s)=\bar{d}^{O U}+\sum_{1<i<N} c_{i} \cdot E_{i} s^{T}$, and the exogenous peer departure rate is $\mu(s)=\bar{d}(s) / F$.

(4) System state: Let $X(s, t)(p(s, t))$ be the number (fraction) of peers in peer state $s$ at time $t$, and $\{X(s, t) \mid s \in \mathcal{S}\}$ be the system state at time $t$. If the total number of peers is $M$, then $X(s, t)=$ $M \cdot p(s, t)$ and $\sum_{s \in \mathcal{S}} p(s, t)=1$. We specify $p(s, t)=0$ for all $s \notin \mathcal{S}$. In the rest of this paper, we omit the term $t$ for simplicity.

Peers can transitions from one peer state to another. For a peer in peer state $s$, it may go to $s+E_{j}, s+E_{j}-E_{k}(j<k)$ when a random request arrives, or go to $s-E_{j}$ when a disconnection occurs, or go to $s-E_{j}+E_{j+1}$ or $s+E_{j}-E_{j+1}$ when the type of a neighbor is changed. Note that when a transition occurs, two or more peers will change their peer states. To simplify the analysis, we assume the peer states of different peers are independent, so we can consider the transition of a single peer at a time. We now express the transition rates between different system states.

(1) $X(s) \rightarrow X(s)+1$ : It means that an exogenous peer of state $s$ enters the system, so the transition rate is $\lambda(s)$.

(2) $X(s) \rightarrow X(s)-1$ : It means that a peer of state $s$ leaves the system, so the transition rate is $M p(s) \mu(s)$.

(3) $X(s), X\left(s+E_{j}\right) \rightarrow X(s)-1, X\left(s+E_{j}\right)+1$ : It means that a random request arrives and a peer of state $s$ (say peer $a$ ) connects to a peer of type $j$ (say peer $b$ ). Suppose peer $a$ is of type $i$, and peer $b$ is in peer state $s_{b}$. We know that $I_{N} s^{T}<N-1$, and before this random request, 1) if $j<N$, peer $b$ must be of type $j-1$, and the transition rate is $2 M \omega \cdot p(s) \operatorname{Prob}\left\{s_{b} \in \mathcal{S}_{j-1}\right\}$, where $\operatorname{Prob}\left\{s_{b} \in\right.$ $\left.\mathcal{S}_{j-1}\right\}=\sum_{s_{b} \in \mathcal{S}_{j-1}} p\left(s_{b}\right)$, and 2) if $j=N$, peer $b$ must be of type $N-1$ or $N$, and the transition rate is $2 M \omega \cdot p(s) \operatorname{Prob}\left\{s_{b} \in\right.$ $\left.\mathcal{S}_{N-1} \cup \mathcal{S}_{N}, I_{i+1} s_{b}^{T}<N-1\right\}$.

(4) $X(s), X\left(s-E_{j}\right) \rightarrow X(s)-1, X\left(s-E_{j}\right)+1$ : It means that a disconnection occurs and a neighbor of type $j$ terminates the connection with a peer in peer state $s$. Let $s \in \mathcal{S}_{i}$, and then the transition rate is $M p(s) \cdot E_{j} s^{T} \rho(i, j)$. Please refer to Appendix A for the derivation of $\rho(i, j)$.

(5) $X(s), X\left(s+E_{j}-E_{k}\right) \rightarrow X(s)-1, X\left(s+E_{j}-E_{k}\right)+1$ : It means that a random request arrives at a peer of state $s \in \mathcal{S}_{N}$ (say peer $a$ ). Suppose the minimum bandwidth download which peer $a$ receives is from a peer of type $k$ (say $a^{\prime}$ ), so $E_{k} s^{T}>0$ and $I_{k} s^{T}=N-1$. Then peer $a$ terminates the connection with peer $a^{\prime}$, and connects to a peer of type $j$ (say peer $b$ ), where $j<k$. So when $E_{k} s^{T}>0, I_{k} s^{T}=N-1$ and $j<k$ hold, the transition rate is $2 M \omega \cdot p(s) \operatorname{Prob}\left\{s_{b} \in \mathcal{S}_{j-1}, I_{N} s_{b}^{T}<N-1\right\}$.

(6) $X(s), X\left(s-E_{j}+E_{j+1}\right) \rightarrow X(s)-1, X\left(s-E_{j}+E_{j+1}\right)+1$ : It means that for a peer of state $s$, one of the neighbors of type $j$ connects to a new peer, and then becomes a neighbor of type $j+1$, where we know that $2 \leq j \leq N-1$. We denote by $\gamma^{+}(j)$ the rate that a type $j$ peer becomes a type $j+1$ peer, and then the transition rate is $M p(s) \cdot E_{j} s^{T} \gamma^{+}(j)$. Please refer to Appendix B for the derivation of $\gamma^{+}(j)$.

(7) $X(s), X\left(s+E_{j-1}-E_{j}\right) \rightarrow X(s)-1, X\left(s+E_{j-1}-E_{j}\right)+1$ : It means that for a peer of state $s$, one of the neighbors of type $j$ loses a neighbor, and then becomes a neighbor of type $j-1$, where we know $3 \leq j \leq N$. We denote by $\gamma^{-}(i, j)$ the rate that a type $j$ peer becomes a type $j-1$ peer, which has at least one neighbor of type $i$. Then the transition rate is $M p(s) \cdot E_{j} s^{T} \gamma^{-}(i, j)$. Please refer to Appendix $\mathrm{C}$ for the derivation of $\gamma^{-}(i, j)$.

When $I_{N} s^{T}<N-1$, we have:

$$
\begin{aligned}
& \frac{d}{d t} X(s, t)=\lambda(s)-M p(s) \mu(s) \\
& -\sum_{2 \leq j \leq N-1} 2 M \omega \cdot p(s) \operatorname{Prob}\left\{s_{b} \in \mathcal{S}_{j-1}\right\} \\
& -2 M \omega \cdot p(s) \operatorname{Prob}\left\{s_{b} \in \mathcal{S}_{N-1} \cup \mathcal{S}_{N}, I_{i+1} s_{b}^{T}<N-1\right\} \\
& +\sum_{2 \leq j \leq N-1} 2 M \omega \cdot p\left(s-E_{j}\right) \operatorname{Prob}\left\{s_{b} \in \mathcal{S}_{j-1}\right\} \\
& +2 M \omega \cdot p\left(s-E_{N}\right) \operatorname{Prob}\left\{s_{b} \in \mathcal{S}_{N-1} \cup \mathcal{S}_{N}, I_{i} s_{b}^{T}<N-1\right\} \\
& -\sum_{2 \leq j \leq N} M p(s) \cdot E_{j} s^{T} \rho(i, j) \\
& +\sum_{2 \leq j \leq N} M p\left(s+E_{j}\right) \cdot\left(E_{j} s^{T}+1\right) \rho(i+1, j) \\
& -\sum_{2 \leq j \leq N-1} M p(s) \cdot E_{j} s^{T} \gamma^{+}(j) \\
& +\sum_{3 \leq j \leq N} M p\left(s+E_{j-1}-E_{j}\right) \cdot\left(E_{j-1} s^{T}+1\right) \gamma^{+}(j-1) \\
& -\sum_{3 \leq j \leq N} M p(s) \cdot E_{j} s^{T} \gamma^{-}(i, j) \\
& +\sum_{2 \leq j \leq N-1} M p\left(s-E_{j}+E_{j+1}\right) \cdot\left(E_{j+1} s^{T}+1\right) \gamma^{-}(i, j+1)
\end{aligned}
$$

Similarly, we can get $\frac{d}{d t} X(s, t)$ when $I_{N} s^{T}=N-1$. At the steady state, we have $\frac{d}{d t} X(s, t)=0$, and we can get an equation for each $s \in \mathcal{S}$. Note that all other parameters can be expressed by $\{p(s) \mid s \in \mathcal{S}\}$, so we can get $\{p(s) \mid s \in \mathcal{S}\}$ by solving this system of equations. As a result, we can compute the fractions of peers with different connection numbers and then the average number of connections per peer at steady state.

\section{SIMULATION RESULT}

We carry out simulation to verify the proposed model. In the simulations, we let $C=1000 \mathrm{Kpbs}, N=4, \lambda=1, \omega=1 / 30$, and vary the file size. Each simulation is executed for 5000 or 10000 seconds, and the fractions of peers with different connection 
numbers $\left\{q_{i} \mid 1 \leq i \leq N\right\}$ are depicted in Fig.1, Fig.2 and Fig.3. We also solve the system of equations from our model by GaussNewton method. After getting $\{p(s) \mid s \in \mathcal{S}\}$, we compute $\left\{q_{i} \mid 1 \leq\right.$ $i \leq N\}$, whose values are also plotted in the corresponding figures.

We can observe that the numerical results from our model match well with the simulation results, especially when the file size is large. Moreover, from Fig.1, we know that only about $15 \%$ peers can connect with enough neighbors, and we can compute the average number of connections per peer, which is less than 1.5. From Fig. 2 and Fig.3, we know that more than $70 \%$ peers can connect with enough neighbors, and we can also compute the average numbers of connections per peer, which are more than 3.5. So the average number of connections per peer increases with the file size.
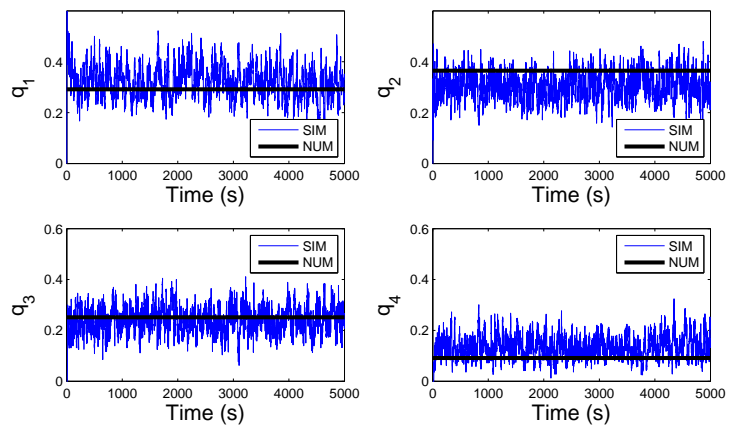

Figure 1: File size is $10 M$.
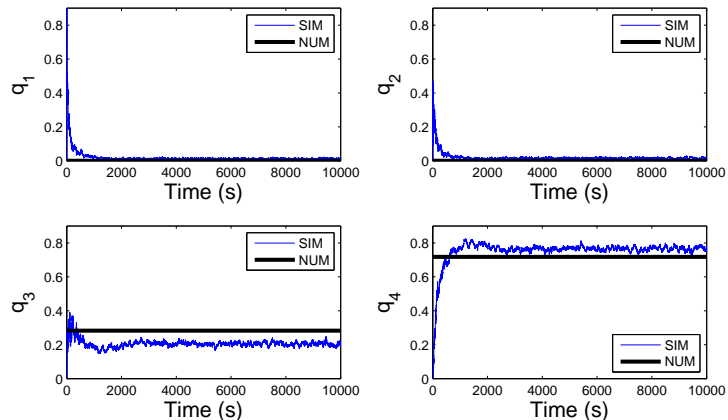

Figure 2: File size is $100 M$.
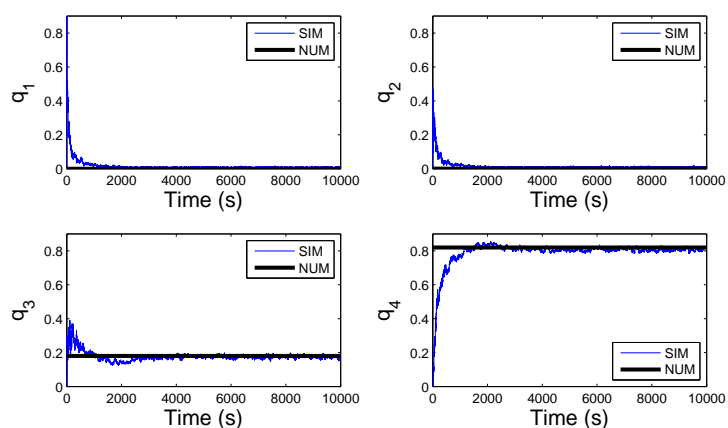

Figure 3: File size is $300 M$.

\section{CONCLUSION}

In this paper, we study the dynamics of BT systems, and propose a stochastic model to evaluate the average number of connections per peer at steady state. We observe that this value increases with the file size. Using this model, BT users can choose the maximum number of connections according to the file size to minimize their costs. For future work, we plan to extend this model to study heterogeneous peers, and investigate the performance gains for peers with different upload capacities. This can help us to understand the behavior of peers which are able to adjust the upload capacities.

\section{REFERENCES}

[1] Bittorrent protocol. http://www.bittorrent.com/protocol.html.

[2] B. Fan, J. C. S. Lui, and D. M. Chiu. The delicate tradeoffs in designing bittorrent-like file sharing protocols'. To appear in IEEE/ACM Transcations on Networking.

[3] W. C. Liao, F. Papadopoulos, and K. Psounis. Performance analysis of bittorrent-like systems with heterogeneous users. Performance Evaluation, 64(9-12), 2007

[4] M. H. Lin, B. Fan, D. M. Chiu, and J. C. Lui. Stochastic analysis of file swarming systems. Performance Evaluation, 64(9-12), 2007.

[5] J. Mundinger, R. R. Weber, and G. Weiss. Analysis of peer-to-peer file dissemination amongst users of different upload capacities. SIGMETRICS Performance Evaluation Review, 34(2):5-6, 2006.

[6] D. Qiu and R. Srikant. Modeling and performance analysis of bittorrent-like peer-to-peer networks. In Proc. ACM SIGCOMM, Portland, Oregon, USA, August 2004.

\section{APPENDIX}

\section{A. DERIVATION OF $\rho(I, J)$}

From equation (1), we can specify that $\rho(i, j)=\mu_{j}+\sigma(i, j)$, where $\sigma(i, j)$ is the rate of a type $j$ peer (say peer $b$ ) terminating the connection with a type $i$ peer (say peer $a$ ) since it finds a better neighbor (say peer $c$ ). We know that if $i \leq 2$ or $j<N, \sigma(i, j)=$ 0 . Otherwise,

$$
\begin{array}{r}
\sigma(i, j)=\sum_{\substack{E_{i} s_{b}^{T}>0 \\
I_{i} s_{b}^{T}=N-1}} 2 \omega \frac{p\left(s_{b}\right)}{\operatorname{Prob}\left\{s \in \mathcal{S}_{N}, E_{i} s^{T}>0\right\}} \\
\cdot \operatorname{Prob}\left\{I_{N} s_{c}^{T}<i-1\right\} \frac{1}{E_{i} s_{b}^{T}} .
\end{array}
$$

So we can get $\rho(i, j)$.

\section{B. DERIVATION OF $\gamma^{+}(J)$}

Suppose a random request arrives at a peer of type $j(j<N)$, and the other peer involved is of type $s$. We know that if $I_{j+1} s^{T}<$ $N-1$, a new connection will be established. So

$$
\gamma^{+}(j)=2 \omega \operatorname{Prob}\left\{I_{j+1} s^{T}<N-1\right\} .
$$

\section{DERIVATION OF $\gamma^{-}(I, J)$}

Note that, for a peer of type $j$, the rate of a type $k$ neighbor terminating the connection is $\rho(j, k)$. Let this type $j$ peer be in peer state $s$, we know that if $k=i, E_{i} s^{T}>1$, and if $k \neq i$, $E_{i} s^{T}>0$. So we can get

$$
\begin{aligned}
\gamma^{-}(i, j) & =\sum_{E_{i} s^{T}>1, s \in \mathcal{S}_{j}} \frac{p(s)\left(E_{i} s^{T}-1\right) \rho(j, i)}{\operatorname{Prob}\left\{E_{i} s^{T}>0, s^{\prime} \in \mathcal{S}_{j}\right\}} \\
+ & \sum_{\substack{2 \leq k \leq N, k \neq i \\
E_{i} s^{T}>0, s \in \mathcal{S}_{j}}} \frac{p(s) E_{k} s^{T} \rho(j, k)}{\operatorname{Prob}\left\{E_{i} s^{\prime T}>0, s^{\prime} \in \mathcal{S}_{j}\right\}}
\end{aligned}
$$

